\title{
EVALUACIÓN DE DOS ATRIBUTOS DE CALIDAD CRÍTICOS EN \\ LA PRODUCCIÓN DE FORMAS FARMACÉUTICAS SÓLIDAS UTILIZANDO LA ESPECTROSCOPIA DE INFRARROJO CERCANO
}

\author{
Ruben Cueva Mestanza*a
}

\begin{abstract}
RESUMEN
La determinación mediante espectroscopia de infrarrojo cercano de atributos críticos de calidad, de interés en la fabricación industrial de sólidos farmacéuticos, es estudiada con el objetivo de aplicar esta nueva metodología más rápida y eficazmente como una alternativa a los métodos convencionales. Se estudia el proceso de mezclado de los componentes de una fórmula efervescente con el fin de determinar el tiempo final del mezclado de una forma rápida y confiable, así como la humedad en los granulados intermedios de tabletas masticables. El objetivo es construir la calidad del producto final, controlando atributos críticos de calidad de los productos intermedios en el mismo proceso aplicando la Tecnología Analítica de Procesos (PAT).
\end{abstract}

Palabras claves: espectroscopia de infrarrojo cercano, atributos críticos, comprimidos farmacéuticos, Tecnología Analítica de Procesos (PAT).

\section{EVALUATION OF TWO CRITICAL QUALITY ATTRIBUTES IN THE PRODUCTION OF SOLID DOSAGE FORMS USING NEAR INFRARED SPECTROSCOPY}

\begin{abstract}
The determination by means of near infrared spectroscopy of critical quality attributes, of interest for the industrial manufacture of pharmaceutical solids, is studied here in order to apply this new methodology more quickly and efficiently as an alternative to conventional methods. The mixing process of the components of an effervescent formula is studied in order to determine the final mixing time in a fast and reliable way, as well as the humidity in intermediate granules of chewable tablets. The objective is to build the quality of the final product, controlling critical quality attributes of intermediate products during the same process by applying Process Analytical Technology (PAT).
\end{abstract}

Key words: near infrared spectroscopy, critical attributes, pharmaceutical solids, Process Analytical Technology (PAT).

*a Universidad María Auxiliadora. eduardo5121@hotmail.com 


\section{INTRODUCCIÓN}

Las formas de dosificación sólidas son las formulaciones farmacéuticas mayormente prescritas. Estas consisten en una mezcla homogénea de ingredientes que proveen las características deseadas a la forma farmacéutica sólida. La mezcla es una etapa crucial en el proceso de fabricación de medicamentos en forma sólida. La mezcla es también un proceso complejo e influenciado por muchas variables. Por ejemplo, las características de los sólidos o del recipiente donde se ha de colocar la mezcla y las condiciones de funcionamiento del mezclador $^{1,2}$. La uniformidad de la dosificación de las formas farmacéuticas se controla normalmente con la toma de muestras en diferentes etapas del mezclado. El principio activo contenido de estas muestras se determina por métodos laboriosos y costosos. De hecho, la mayor parte del tiempo que se consume en el proceso de mezcla no es la operación de mezcla en sí, sino los análisis para confirmar el punto final de la mezcla. Por lo tanto, la espectroscopia de infrarrojo cercano (NIRS) se presenta como una alternativa a los métodos anteriores debido a que no requieren ningún tratamiento previo y la información se puede obtener durante el proceso. A diferencia de los métodos convencionales de análisis, la espectroscopia NIR es rápida, no invasiva y proporciona información acerca de las propiedades físicas y químicas de la muestra ${ }^{2}$.

Otra etapa crítica es el proceso de granulación, el cual es utilizado para el incremento del tamaño de partícula que permite obtener una alimentación uniforme de las máquinas de compresión y un llenado reproducible de la matriz de los comprimidos, lo que se traduce en una compactación uniforme de las partículas, asegurando así la uniformidad del peso de los comprimidos y unas propiedades físico-mecánicas constantes (dureza, friabilidad, etc.). Las características que afectan a la fluidez de un polvo o granulado son: el tamaño de partícula medio, la distribución del tamaño de partícula, la forma de las partículas, la rugosidad de la superficie y el contenido de humedad. La humedad de una muestra influye notablemente en la fluidez de los polvos de la misma forma que en el proceso de compactación. El agua tiende a formar aglomerados no fluentes y si es excesiva se tiende a pegar en las paredes y reducir la fluidez del granulado. En la compactación, las tabletas con porcentajes de humedad altos tenderán a adherirse a la matriz, forzando la eyección, produciendo tabletas rotas y/o laminadas. Por otro lado, si se tiene granulados demasiado secos, esto afectará tanto al aspecto de la tableta y a la disolución de esta ${ }^{3,4}$.

Los métodos para determinar el punto final en el mezclado y humedad puede afectar el tiempo de producción al detener el proceso productivo por el traslado de la muestra y la obtención de resultados. Por tal motivo, métodos rápidos en el mismo proceso reducirán el ciclo productivo del proceso, aumentando la productividad y a su vez asegurando la calidad de producto. El objetivo general de este trabajo es investigar la aplicación de la espectroscopia NIR para evaluar la calidad del producto a partir de dos atributos críticos de calidad en dos etapas: el mezclado (homogeneidad) y la granulación (humedad), que tienen un gran impacto en el producto final y de este modo alcanzar un mejor conocimiento del proceso y de los puntos críticos que pueden afectarlos. Este estudio se divide en dos partes. La primera examina la aplicación de la espectroscopia NIR para determinar el punto final del mezclado, donde la 
mezcla se considera homogénea en un polvo efervescente, y la segunda examina el uso de la espectroscopia NIR para predecir humedad en los granulados. Estas metodologías son desarrolladas con el fin de ser aplicadas para entender y controlar el proceso de mezclado y granulación de formas farmacéuticas sólidas sin afectar los tiempos de fabricación.

\section{PARTE EXPERIMENTAL}

\section{Materiales y métodos}

Proceso de mezclado y toma de muestra. El proceso de mezclado de los componentes del polvo efervescente se realiza en un mezclador en $\mathrm{V}$ con un tiempo de mezclado de 30 minutos. Se tomaron seis muestras a diferentes tiempos durante el proceso, a los 10, 20 y 30 minutos (figura 1) y al inicio, mitad y al final de la descarga del mezclador.

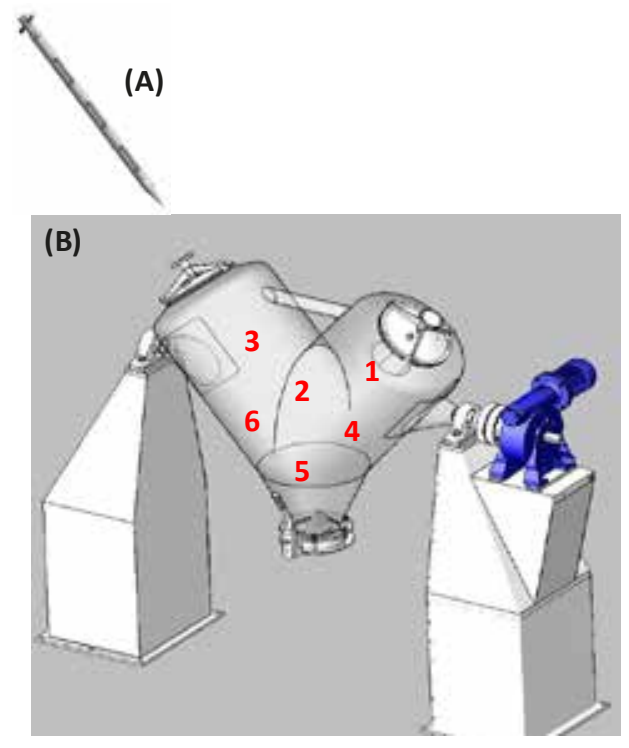

Figura 1. (A) Muestreador utilizado para la toma de muestras (B) Mezclador en V donde se remarca los seis puntos para el muestreo durante el mezclado.

Proceso de secado y toma de muestra. El secado de los granulados intermedio de las tabletas masticables se ha realizado en un secador de lecho fluido. A continuación se muestra las etapas del proceso y las muestras que se toman en cada una de ellas:

- Granulación húmeda (se toma muestras de los granulados húmedos antes del proceso de secado)

- $\quad$ Secado (se toma muestra a los 15, 30, 45 minutos y al final del secado)

- Granulación seca (se tomaron muestras de los granulados obtenidos a partir de la molienda y tamizado por malla de $300 \mu \mathrm{m}$ de diámetro de poro, de los granulados obtenidos en la fase de secado) 
Hardware y software. Los espectros de reflectancia fueron adquiridos con un espectrofotómetro NIR con analizador de mano PHazir TM. (Polychromix, Inc.) controlado a través del software Polychromix Phazir TM MG; cada espectro fue el resultado de 5 scans. En la figura 2 se muestra el equipo utilizado. Los espectros se registraron en un rango de longitudes de onda de 1600-2400 nm con una resolución de $12 \mathrm{~nm}$. El software Unscrambler V. 9.8 de Camo Process (Trondheim, Noruega) fue utilizado para el tratamiento matemático.

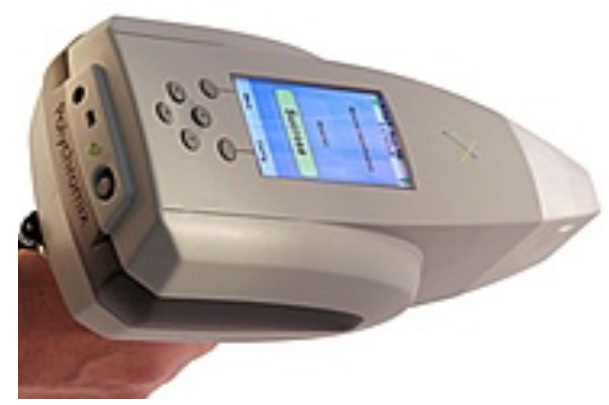

Figura 2. Analizador NIR de mano PHazir TM (foto)

Registro de los espectros. Los espectros se registraron a través de la bolsa de plástico donde se recogieron las muestras del proceso de mezclado y secado, en el un rango de 1600-2400 $\mathrm{nm}$, usando como referencia una placa de cerámica presente en la tapa de referencia del equipo.

Pre-tratamientos de los espectros NIR. Con el objetivo de reducir la variabilidad asociada a las características físicas o ruido de los espectros obtenidos de la fase de mezclado y secado, los espectros fueron sometidos a diferentes pre-tratamientos espectrales, como son: Estándar Normal Variate (SNV), 1era y 2da derivada utilizando en estas últimas el algoritmo de Savitzky-Golay con una ventana móvil de tres puntos y ajustados a un polinomio de $2^{\text {do }}$ grado.

\section{Metodología cualitativa para determinar la homogeneidad}

- Desviación estándar de espectros. Consiste en el cálculo de la desviación estándar de la absorbancia, DS, a cada longitud de onda de los espectros obtenidos a un determinado tiempo en el proceso de mezclado (Ec. 1). Donde $n$ es el número de espectros ( $n=6), \bar{A}$ es el promedio de las absorbancia a cada longitud de onda de $\mathrm{n}$ espectros consecutivos y $\mathrm{Aj}$ es la absorbancia a cada longitud de onda de cada espectro $\mathrm{j}$.

$$
\text { S.D. }=\sqrt{\frac{\sum_{j=1}^{n}\left(\bar{A}-A_{j}\right)^{2}}{n-1}} \text { (Ec. 1) }
$$

Después se calcula el promedio de S.D. para todas las longitudes de onda, , según (Ec. 2). Donde $\mathrm{m}$ es el número de longitudes de onda i. 


$$
\left.\overline{\mathrm{X}}=\frac{\sum_{i=1}^{\mathrm{m}} \mathrm{S}}{\mathrm{m}} \quad \text { (Ec. } 2\right)
$$

\section{Metodología cuantitativa para determinar humedad en el secado 1.-Método de referencia \\ El método de referencia es el análisis por pérdida por secado en un analizador de Humedad Mettler Toledo Modelo HB43-S.}

\section{2.-Muestras para crear el método para cuantificar la humedad}

La ecuación de calibración PLS1, para cuantificar la humedad, se establece con muestras de producción y muestras de laboratorio (muestras de producción a la cuales se las ha adicionado agua para incrementar su porcentaje de humedad).

Las muestras de laboratorio se prepararon adicionándole agua a granulados de producción con alrededor $2 \%$ de humedad para obtener muestras con aproximadamente 10, 8,5 y 3 $\%$ de humedad. Se comprobó la humedad por el método tradicional/referencia (balanza de humedad) ${ }^{6}$.

El conjunto de muestras (muestras de laboratorio y muestras de producción) se dividen en dos grupos. El primer grupo, conjunto de calibración, se emplea para construir el modelo de calibración. El segundo grupo, conjunto de validación, se utiliza para validar la ecuación de calibración PLS1 y determinar su capacidad predictiva.

\section{3.- Modelo PLS}

El rango espectral empleado para construir el modelo PLS1, para la cuantificación de humedad en granulados de una fórmula para tabletas masticables, fue entre 1844-2009 nm, ya que en este rango tiene mayor absorbancia el agua (figura 3).

El pre-tratamiento matemático de los espectros seleccionados permite la corrección de la derivada de la línea base como consecuencia de la variabilidad habitual en las propiedades físicas de las diferentes muestras, ya que el espectro NIR depende tanto de las propiedades químicas de la muestra como de algunas de sus propiedades físicas, como son la forma, el tamaño de partícula o densidad del polvo. El conjunto de muestras de calibración debe recoger todas las fuentes de variabilidad posibles, por ello es necesario incorporar muestras de laboratorio y granulados de producción para incorporar al modelo la variabilidad debida al proceso de fabricación. El modelo ha sido validado para asegurar su consistencia, usando espectros de muestras de laboratorio y muestras de producción ajenas a las utilizadas para crear el modelo. En este paso se verifican y definen las longitudes de onda a emplear, el número de componentes PLS necesarios y la semejanza de los resultados con los resultados obtenidos por el método de referencia y otros parámetros del modelo PLS1. 


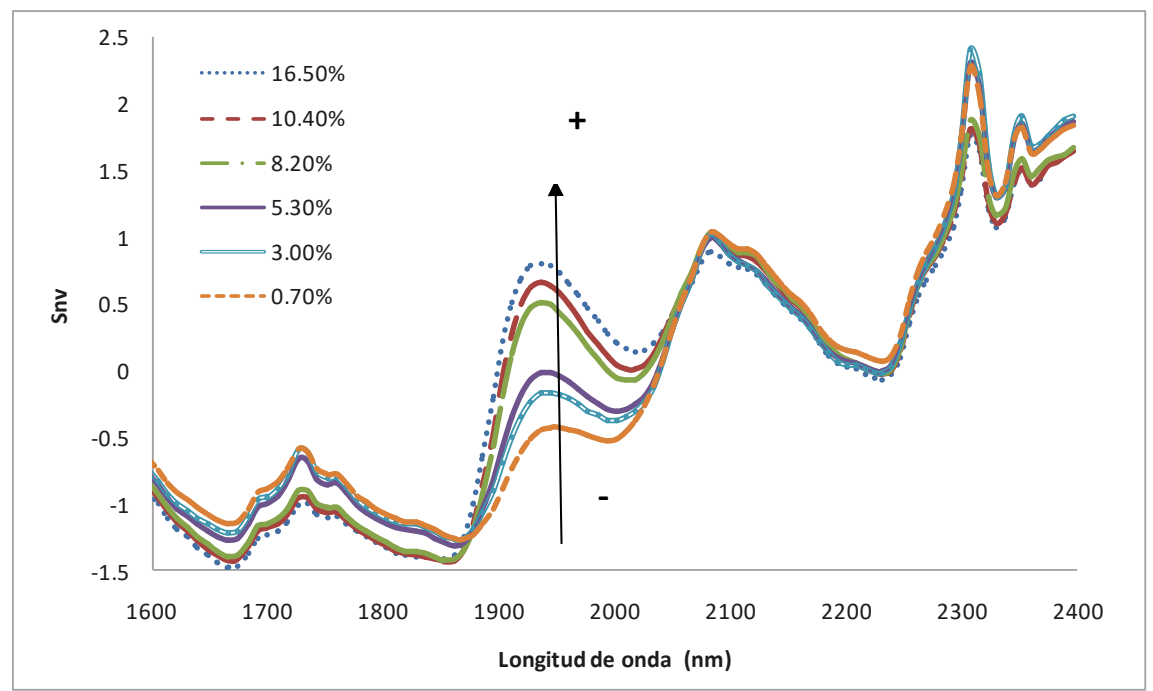

Figura 3. Espectros de muestras con distinto porcentaje de humedad.

El número de factores para el modelo se seleccionó a partir del error estándar relativo (Relative Standard Error, RSE) seleccionando el modelo con menor valor de RSE para el conjunto de predicción.

$$
R S E=\sqrt{\frac{\sum_{i=1}^{m}\left(Y_{i}^{N I R}-Y_{i}^{R E F}\right)^{2}}{\sum_{i=1}^{m} Y_{i}^{R E F^{2}}}}>
$$

La tabla 1 muestra los parámetros más significativos del modelo PLS construido. Aunque el primer factor PLS describe un $97 \%$ de la varianza total, fue necesario dos factores (99\%) para ajustar correctamente el modelo y conseguir una buena capacidad predictiva. 
Tabla 1. Parámetros relevantes de los modelos de calibración construidos para la determinación de la humedad en granulados intermedios para ttabletas masticables.

\begin{tabular}{ccc}
\hline & & Humedad \\
\hline Muestras & Calibración & Predicción \\
Pre-tratamiento & Laboratorio + Producción & Laboratorio + Producción \\
Rango (nm) & & $1844-2009$ \\
Rango de humedad & $16.8-0.7 \%$ & $16.5-0.8 \%$ \\
Factores & 2 & - \\
Varianza explicada Y (\%) & 99.0 & 33 \\
$N^{0}$ muestras & 19 & 1.18 \\
RSEC/P & 0.44 & \\
\hline
\end{tabular}

\section{RESULTADOS Y DISCUSIÓN}

\section{Método cualitativo para determinar el punto final en el mezclado}

\section{1.- Determinación del pre-tratamiento}

Para determinar los pre-tratamientos más idóneos se aplicaron varios tratamientos a cada uno de los espectros obtenidos con el espectrofotómetro NIR del proceso de mezclado, obteniéndose que el pre-tratamiento que reduce más la variabilidad física de la muestra y la producida por el sistema de registro, así como el ruido instrumental, fue el de la $2^{\text {da }}$ derivada.

\section{2.- Determinación cualitativa de la homogeneidad de la mezcla}

Previo a la aplicación de la metodología de desviación estándar de los espectros para la determinación cualitativa de la homogeneidad, se ha comprobado experimentalmente la desviación estándar (DS) de una mezcla que se considera homogénea, donde las variaciones se deben a las variaciones producidas por el registro de los espectros y por el equipo NIR, registrando los espectros de una muestra representativa de la mezcla (mezcla de la parte inicial, media y final de la descarga) la cual ha sido homogenizada manualmente. Se registró seis veces colocando la bolsa de plástico que contiene la mezcla en diferentes posiciones cada vez que se registraba el espectro de la mezcla, obteniéndose un valor de desviación estándar de 0,0012 para los espectros obtenidos. Este valor se toma como valor objetivo para determinar cuando la mezcla es homogénea. La representación gráfica de desviación estándar versus tiempo de muestreo nos permite determinar a qué tiempo se considera la mezcla homogénea, observándose que a los 10 y 20 minutos la mezcla no ha logrado la homogeneidad debido a que la desviación de los espectros es superior de lo que se espera para una mezcla homogénea. Por otro lado, la mezcla a los 30 minutos y en la etapa de la descarga sí se puede considerar homogénea ya que sus valores de DS se encuentran por debajo del valor objetivo. 


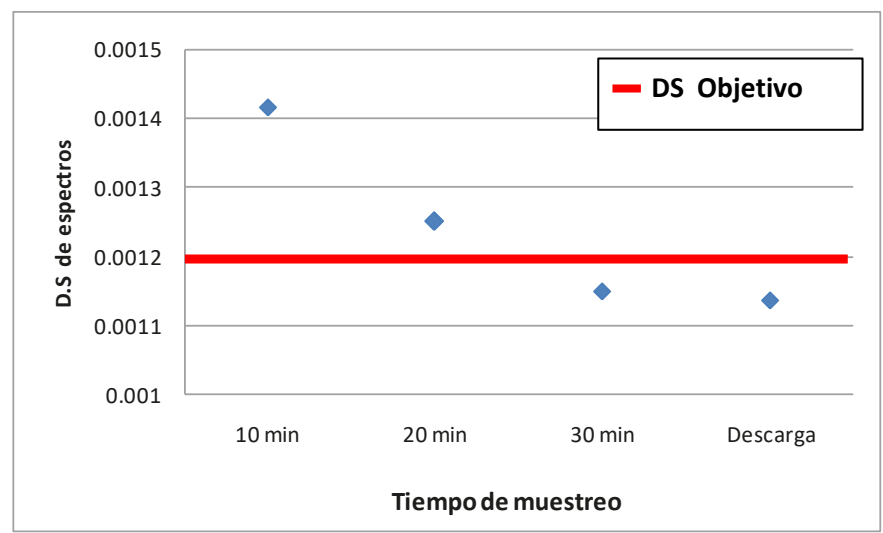

Figura 4. Aplicación de la metodología de la DS en espectro a un proceso de mezclado.

\section{Determinación de la humedad en el proceso de secado}

Los resultados que se presentan corresponden al estudio de secado de granulado intermedio de tabletas masticables. La figura 5 muestra los espectros recogidos durante el proceso de secado, donde se observa la variación en las bandas de absorción durante las etapas del proceso de secado. Se destaca la mayor altura de la banda en la región alrededor de 1950 nm., debido al alto porcentaje de agua antes del secado (granulado húmedo) y su posterior disminución durante el secado del granulado. La importante señal de absorción en la región de las longitudes entre 2250-3260 nm. es debido, principalmente, a la absorción del plástico de la bolsa de muestreo.

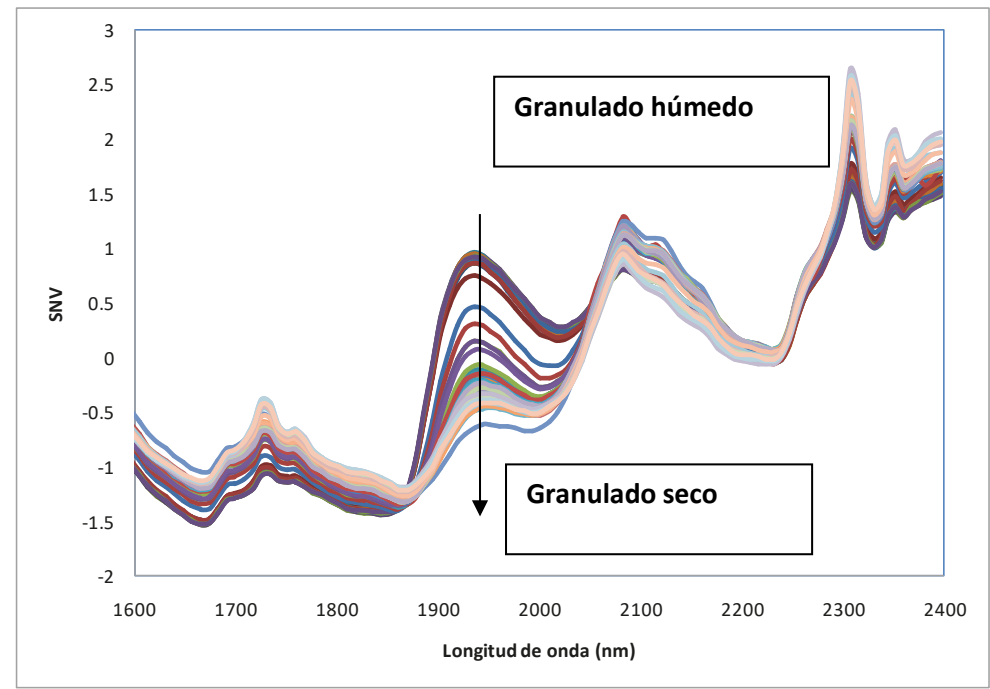

Figura 5. Espectros NIR en un proceso de secado (Pre-tratamiento SNV) 
Un PCA de la matriz de los espectros de un proceso de secado en modo SVN muestra que los dos primeros factores $\mathrm{PC}$ recogen el $99 \%$ de la varianza de todo el proceso.

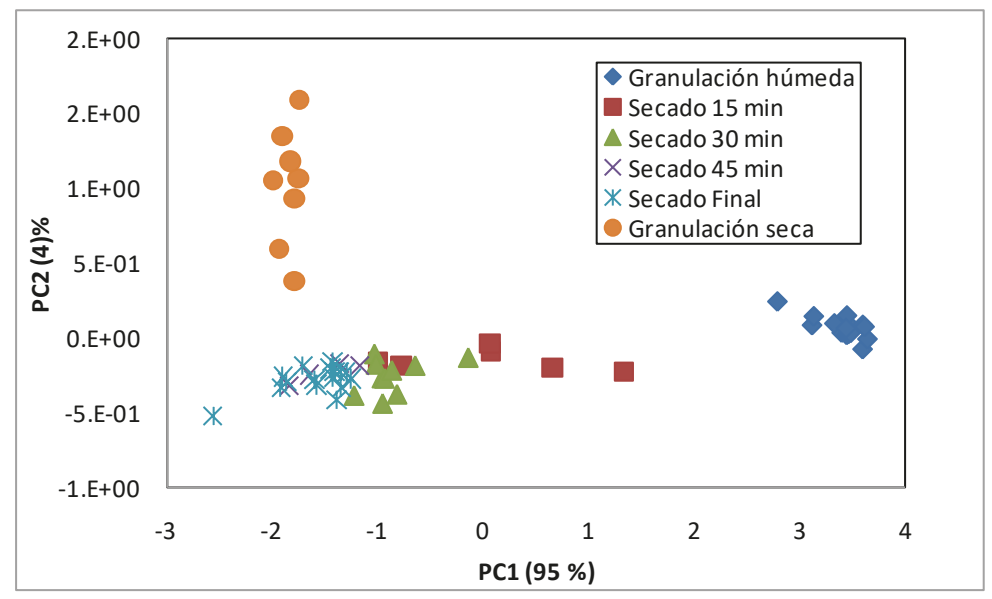

Figura 6. Gráfico de dispersión de una PCA de los espectros de un proceso de secado en modo SNV. Los scores están representados en función de la etapa correspondiente.

En la figura 6 se ha representado el gráfico de dispersión de los scores PC1 vs PC2 de un proceso en el que se muestra los scores en función de la etapa de proceso. En la parte inferior derecha de esta figura aparecen los scores que corresponden a la etapa de granulación húmeda que están agrupados sin mostrar ninguna evolución. Por otro lado, los scores de las muestras recogidas a los minutos 15 y 30 del secado se presentan dispersos en el eje del PC1. Esto puede deberse a que el secado no es uniforme. Sin embargo, a los 45 minutos y al final del secado se encuentran agrupadas, ya que en estos puntos del secado la humedad es semejante entre las muestras.

En las muestras de la granulación húmeda y secados se observa un desplazamiento en el PC1. Este desplazamiento está relacionado con el porcentaje de humedad de la muestra, ya que es la única variable que afecta el proceso. Mientras que en los scores, las muestras de granulación seca tienen un desplazamiento el PC2. Estas muestras se diferencian de las anteriores en el tamaño de partícula, ya que se han tamizado con un tamiz de $300 \mu \mathrm{m}$ de diámetros de poro. Por lo tanto, se puede decir que la variable relacionada con el PC2 es el tamaño de partícula. En el gráfico se pueden distinguir las tres etapas del proceso: granulación húmeda, secado y granulación seca.

\section{2.-Información cuantitativa}

\section{Seguimiento de la evolución de la humedad}

En el estudio cualitativo se demostró la asociación entre el PC1 y el contenido de humedad de la muestra, y se decidió la construcción de un modelo PLS1 para cuantificar el contenido de humedad durante el proceso. 


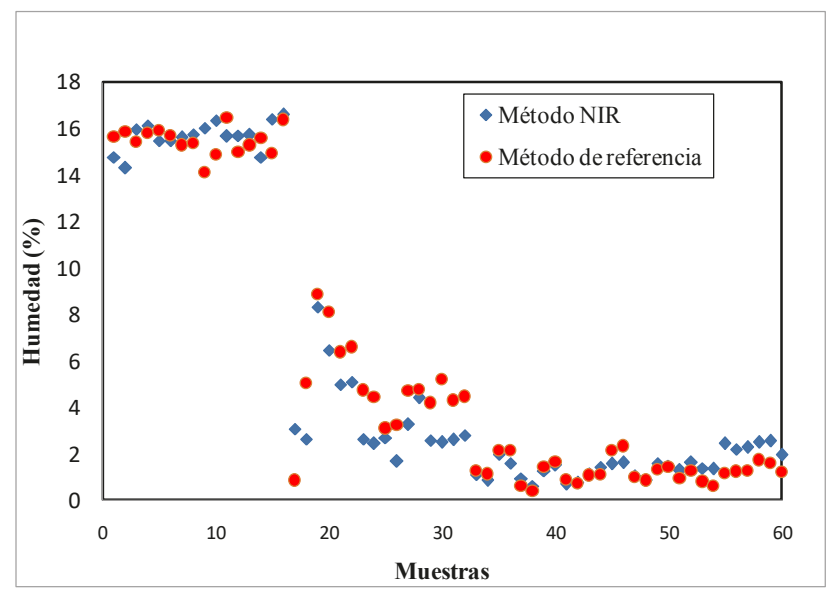

Figura 7. Evolución de los valores del contenido de humedad en un proceso.

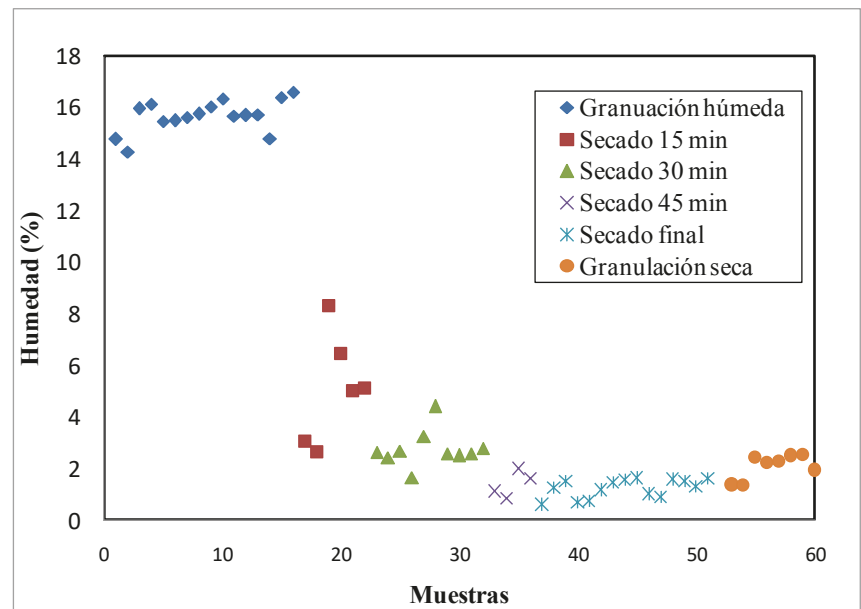

Figura 8. Evolución de los valores del contenido de humedad en un proceso de secado (Método NIR)

La figura 7 muestra la predicción de la evolución del contenido en humedad calculada, con el modelo desarrollado aplicado a un proceso, así como los valores de referencia obtenidos por el método de referencia, como puede apreciarse los resultados obtenidos por los dos métodos son muy semejantes. La figura 8 muestra los resultados obtenidos por el método NIR para las muestras de cada una de las etapas. Las muestras del granulado húmedo se encuentran entre 14 y $17 \%$ de humedad. Por otro lado, se puede observar que a los 15 y 30 minutos del secado se produce la mayor reducción de la humedad, además que los valores de humedad obtenidos en este punto del secado varían, mostrando que el secado no es uniforme. Por otro 
lado, a partir de los 45 minutos la mezcla tiene porcentajes menores a $3 \%$. Sin embargo, se tiene que seguir secando para obtener valores con una desviación reducida y disminuir la humedad del granulado.

\section{CONCLUSIONES}

Este estudio demuestra la aplicación efectiva de NIRS para la determinación de la homogeneidad durante el proceso de mezclado, determinándose el tiempo final del mezclado a los 30 minutos. Se recomienda aplicar este método en fármacos donde el mezclado es crítico en procesos donde, por ejemplo, hay baja concentración del principio activo, los componentes se adhieren a las paredes, o donde las mezclas son susceptibles a su segregación, etc.

Se ha desarrollado una nueva metodología para el seguimiento espectroscópico de un proceso de secado de un granulado en una granuladora de lecho fluido. Los espectros obtenidos han permitido realizar un estudio tanto cualitativo como cuantitativo de la humedad en la fase de secado.

Un análisis multivariante de los espectros NIR mediante un análisis por componentes principales, permite diferenciar las tres etapas del proceso de secado. Los dos primeros componentes principales recogen casi toda la variabilidad de las muestras del proceso y se han identificado las tres etapas del proceso mediante el gráfico de score vs muestras. La interpretación de los loading espectrales permite asociar las fuentes de variabilidad: el primer componente principal explica la variación del contenido de agua, mientras que el segundo componente principal explica la evolución del tamaño de partícula a lo largo del proceso.

El modelo de calibración PLS construido permite realizar la determinación correcta del contenido de humedad.

Se recomienda aplicar esta metodología en casos en que la humedad sea un factor crítico en el proceso, por presencia de productos higroscópicos, por principios activos inestables o porque la humedad afecte la fluidez del granulado o de la compactación.

\section{AGRADECIMIENTOS}

A Laboratorios Medifarma S.A y al D.T. Q.F. Luis Kanashiro, por brindarnos todas las facilidades para la elaboración de esta investigación, cuyos resultados serán aplicados en la empresa. Por otro lado, quisiéramos brindar un agradecimiento especial al área de validaciones de procesos, representada por el Q.F. David Panta y Q.F. Erik Cotera por su apoyo científico y técnico para entender mejor los procesos. 


\section{REFERENCIAS BIBLIOGRÁFICAS}

1. Venables HJ, Wells JI. Powder mixing. Drug Dev Ind Pharm. 2001; 27: 599-612.

2. Ciurczak EW. Pharmaceutical mixing studies using near infrared spectroscopy. Pharm Technol. 1991; 15: 140-145.

3. Liua LX, Marziano I, Bentham AC, Litster JD, White ET, Howes T. Effect of particle properties on the flow ability of ibuprofen powders. Int J Pharm. 2008; 362:109-117.

4. Närvänen T, Lipsanen T, Antikainen O, Räikkönen H, Yliruusi J. Controlling granule size by granulation liquid feed pulsing. Int J Pharm. 2008; 357: 132-138.

5. Rantanen J, Räsänen E, Tenhunen J, Känsäkoski M, Mannermaa J, Yliruusi J. In-line moisture measurement during granulation with a four-wavelength near infrared sensor: an evaluation of particle size and binder effects. Eur J Pharm Biopharm. 2000; 50(2):271276.

6. Kirk RS, Sawyer R, Egan H. Composición y análisis de alimentos de Pearson. Segunda edición. México DF: Compañía editorial continental SA; 1996. 